\title{
Drying and percolation in correlated porous media
}

\author{
Soumyajyoti Biswas, ${ }^{1}$ Paolo Fantinel, ${ }^{1}$ Oshri \\ Borgman, ${ }^{2}$ Ran Holtzman, ${ }^{2}$ and Lucas Goehring ${ }^{3, *}$ \\ ${ }^{1}$ Max Planck Institute for Dynamics and \\ Self-Organisation (MPIDS), Göttingen 3707\%, Germany \\ ${ }^{2}$ Department of Soil and Water Sciences, \\ The Hebrew University of Jerusalem, Israel \\ ${ }^{3}$ School of Science and Technology, \\ Nottingham Trent University, Nottingham, NG11 8NS, UK
}

(Dated: November 22, 2018)

\begin{abstract}
We study how the dynamics of a drying front propagating through a porous medium are affected by small-scale correlations in material properties. For this, we first present drying experiments in micro-fluidic micro-models of porous media. Here, the fluid pressures develop more intermittent dynamics as local correlations are added to the structure of the pore spaces. We also consider this problem numerically, using a model of invasion percolation with trapping, and find that there is a crossover in invasion behaviour associated with the length-scale of the disorder in the system. The critical exponents that describe large enough events are similar to the classic invasion percolation problem, while the addition of a finite correlation length significantly affects the exponent values of avalanches and bursts, up to some characteristic size. We thus find that even a weak local structure can interfere with the universality of invasion percolation phenomena. This has implications for a variety of multi-phase flow problems, such as drying, drainage, and fluid invasion.
\end{abstract}

\footnotetext{
* lucas.goehring@ntu.ac.uk
} 


\section{INTRODUCTION}

The flows of many fluids into porous media have been found to evolve complex shapes with extensive interfaces, which are well described by the fractal morphology of invasion percolation models $[1-5]$. Nonetheless, these flows will also often reflect any microstructure of the materials in which they move, for example travelling along preferential paths like fractures. In fluid-fluid invasion [6-8], and the drying of porous media [9, 10], it has been demonstrated that local correlations in the pore-scale properties of a granular medium can dramatically modify any fluid flows within it. For immiscible flows, as occur when oil displaces water, increasing the correlation length (the distance over which pores are approximately the same size) of pore-scale disorder has been shown to decrease the residual saturation of the wetting phase at breakthrough $[6,7]$, leading to a more gradually-varying capillary pressure-saturation relation [11, 12], and improving the connectivity, and hence permeability, of both phases [12]. Changes in fluid retention have also been observed upon varying the correlation length of particle wettability in a bead pack [8]. These types of flows have a wide range of practical applications, for example involving the production of groundwater and hydrocarbons, monitoring or assessing the contamination of soils and water sources and their remediation, the safe storage of hazardous wastes and carbon sequestration $[13,14]$.

The physics of multi-phase flows often leads naturally to the ideas of intermittent dynamics. Such dynamics are characterised by short bursts of activity that are separated by much longer quiescent (i.e. event-free) intervals, and appear in diverse physical, biological and even social systems [15-18]. For fluids, the classic examples include Haines jumps $[19,20]$, or instabilities triggered by the burst, touch, or overlap of nearby menisci [21, 22]. However, more diverse examples of the same universal phenomenon range from earthquakes [23] to granular avalanches [24], fracture propagation [25], turbulent energy transfer [26], variations in stock markets [27], blackouts in power grids [28], and evolution [29]. Much of the complex behaviour of these systems can be captured by surprisingly simple models. Specifically, the statistics of intermittent dynamics are routinely found to be equivalent to invasion percolation [1], a model of a network of fragile bonds which fail as a system is stressed. In this model a failure front, corresponding to the leading edge of a fluid invasion, reaction or drying front, drives its way through the network, and the activity of this front 

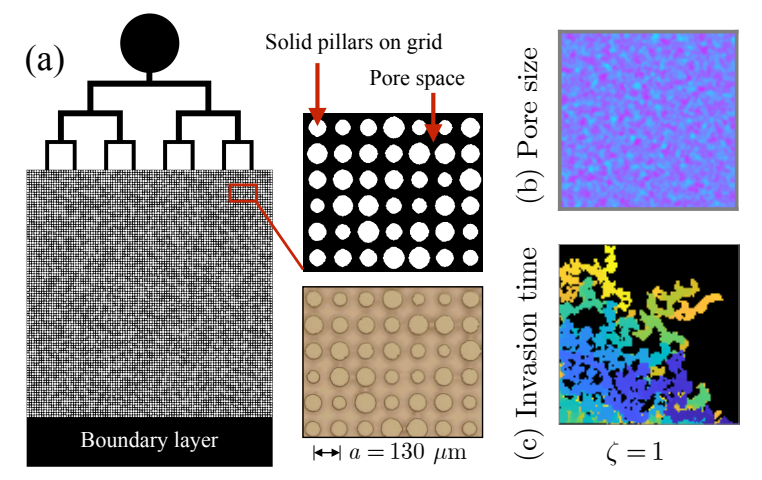

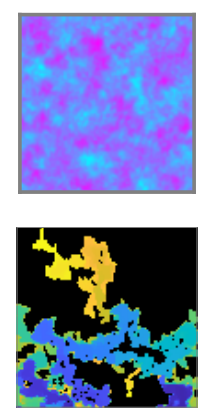

$\zeta=4$

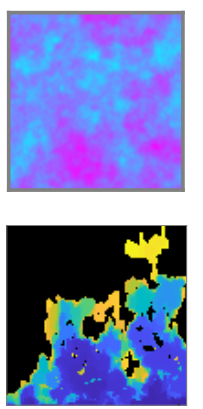

$\zeta=10$
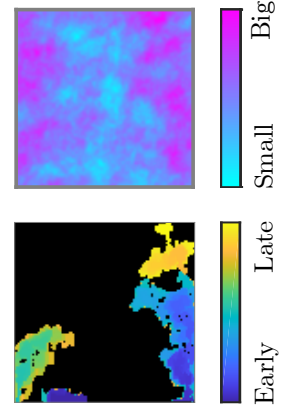

$\zeta=15$

FIG. 1. Invasion into correlated porous media. (a) A flat pore space is designed and fabricated in a micro-fluidic chip by soft lithography techniques. The drying cell consists of an array of pillars, which surround pores. The pores are initially filled through channels along one edge of the cell and then dry through the opposite edge, which is open to the atmosphere. (b) Correlations between pillar sizes, and hence pore sizes, are randomly introduced with different correlation lengths $\zeta$. (c) The drying pattern (shown here at breakthrough, when the invading phase connects to the back of the chip) and sequence of invaded pores reflect the underlying structure of pillar sizes.

is found to obey well-defined and universal scaling laws [2, 30].

Here, we consider how local correlations in the strength of a system can modify the universal response of invasion percolation, and explore this response within a paradigm experimental system, namely a drying porous medium. During drying, evaporation of a defending fluid increases the curvature of any menisci caught in the pore spaces, resulting in the capillary invasion of air, once a local pressure threshold is exceeded. We study, both experimentally and numerically, the invasion patterns and statistics of a drying front moving through such a disordered porous medium, where the sizes of nearby pores are correlated with each other. The experimental realisation is a quasi-two-dimensional micro-fluidic chip, where fluids can move around an array of rigid pillars arranged on a regular grid [10], as described in Fig. 1. The pillar radii are correlated over a given length scale $\zeta[9,10]$. The numerical simulation is that of invasion percolation on a grid, with its parameters taken from the geometry of the experiments. In both cases, as shown in Fig. 2, the capillary pressure at the drying front fluctuates, and spatial correlations change the statistics of these fluctuations, such as the likelihoods of extreme pressures, or the distributions of sudden bursts of activity.

Our primary aim is to explore and explain the effects of such a correlation length, interme- 

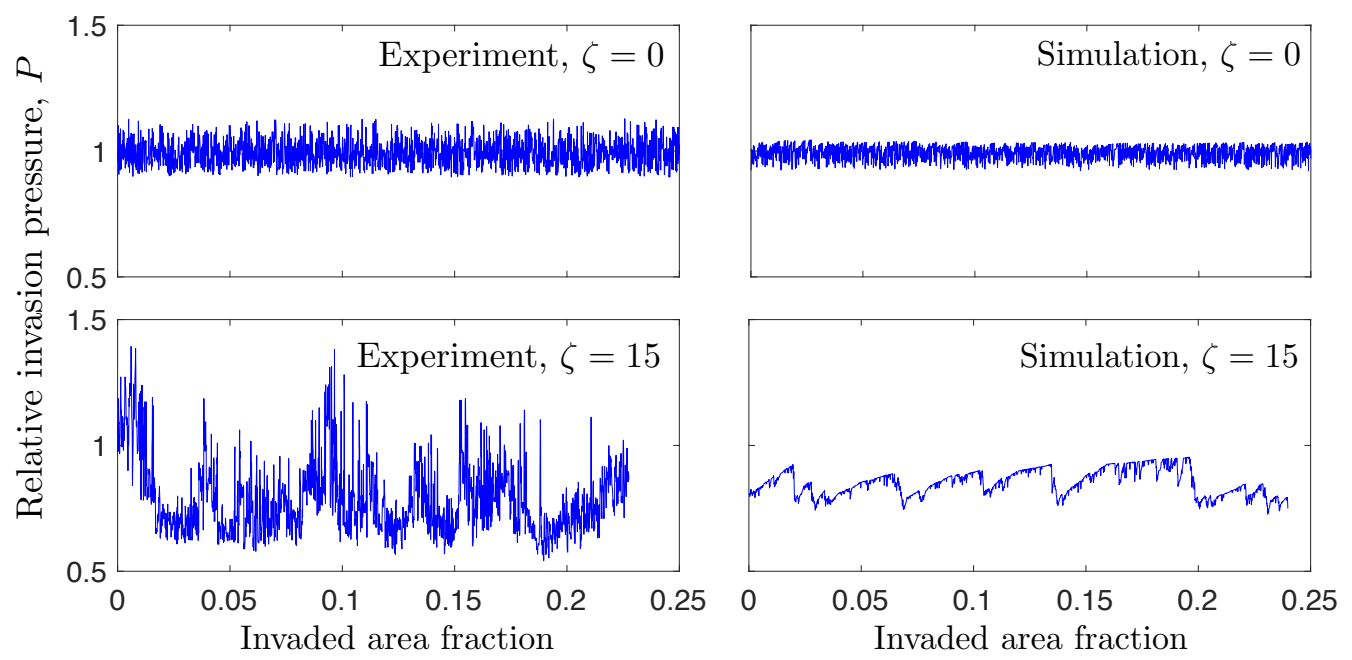

FIG. 2. Local correlations in the sizes of pores and throats dramatically affect how the fluid pressure fluctuates as a porous medium is dried, in both experiments and a complimentary invasionpercolation model. When there are no correlations $(\zeta=0)$ the pressure sequence is similar to white noise, centred around the average invasion pressure of the porous medium $(P=1)$. In contrast, when the correlation length is $\zeta=15$ times the typical pore size, the pressure sequence shows strong intermittency.

diate between the pore or grid-scale and the system size, on fluid invasion phenomena. Given that the intermittent dynamics of the drying front is a critical phenomenon, its statistics can be characterised by a set of critical exponents [4, 5, 31, 32]. We find that these exponents are changed by the introduction of the correlation length scale. Connecting these features directly to our micofluidic experiments, we further investigate these effects and determine the scaling relations for drying interfaces in correlated media.

\section{METHODS}

\section{A. Microfluidic experiments}

Experiments were conducted in micro-fluidic chips containing a mechanical micro-model of a porous medium; detailed methods of their design and fabrication are given in Refs. $[9,10]$. Briefly, we used soft lithography techniques to create a pattern of $100 \times 100$ solid pillars on a regular grid, of spacing $130 \mu \mathrm{m}$, defining a pore space between them. The pillars had an average radius of $a=50 \mu \mathrm{m}$ and height $h=40 \mu \mathrm{m}$ and the pore space was 
designed in a Hele-Shaw geometry, as in Fig. 1(a). To introduce disorder, the pillar sizes were randomised over the range of $40-60 \mu \mathrm{m}$. For samples with no correlations, the size of each pillar was selected randomly from a uniform distribution over this range. To make geometries with correlations in pillar sizes, such as patches of larger or smaller pillars, we generated random Gaussian rough surfaces $[9,33]$ with a characteristic correlation length $\zeta$. Pillar sizes were then assigned by sampling this surface at the grid positions, and mapping the resulting values onto the range of $40-60 \mu \mathrm{m}$. The manufacturing tolerance on these pillar sizes is estimated at about $\pm 3 \%$ [10]. The length $\zeta$ defines the distance, measured in grid spacings, over which nearby pillars will have roughly similar sizes. Example designs are shown in Fig. 1(b).

The system was then filled with a wetting volatile oil and allowed to dry from one edge of the cell. The drying pattern was imaged by an overhead camera, and the time-lapse images were used to find the time at which any given pore was invaded by air, as in Fig. 1(c). By identifying the widest exposed throat on each invaded pore, at the moment of its invasion, the sequence of pore invasions was used to determine the sequence of invasion pressures, during drying. We consider the absolute value of the pressure sequence, for simplicity, although the actual capillary pressure will be negative (i.e. lower than atmospheric). These

pressures were then normalised by the characteristic pressure $\bar{P}=2 \gamma(1 / w+1 / h)$, where $w=30 \mu \mathrm{m}$ is the average throat width, and $\gamma$ is the surface tension. In this study we focussed on the relative pressure, $P$, of the main cluster, which is the set of filled pores that remain connected to the rear of the chip. As shown in Fig. 2, the observed pressure fluctuations of the main cluster show intermittent dynamics, which depend on the range of correlations in the experiment.

\section{B. Invasion percolation model}

In order to simulate these dynamics we used a minimal model of the drying front i.e. the fluid-air interface of the main cluster. The pore throats each have a threshold pressure (again, taking the absolute value, relative to atmospheric pressure), which must be exceeded in order to invade a pore through that throat. As in the experiments, we considered a square lattice of pillars, using the same algorithms for randomly selecting the pillar sizes, with correlation length $\zeta$. The separations between adjacent pillars give the 

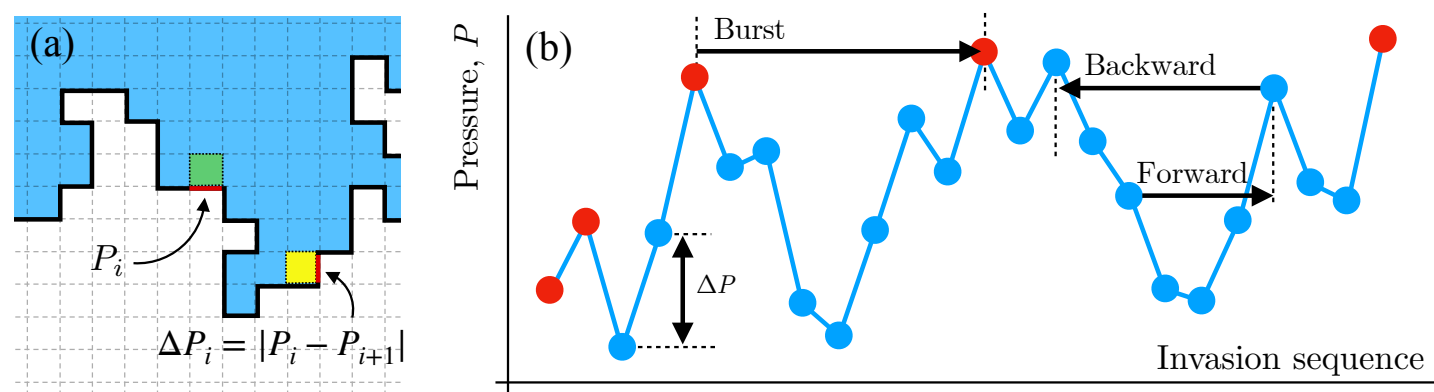

FIG. 3. Model and statistics of the pressure-saturation fluctuations. (a) The invasion-percolation model tracks an interfacial line between wet (blue) and unsaturated (white) regions. At every time step $i$ the interfacial throat with the lowest absolute invasion pressure $P_{i}$ is broken, and air invades the pore behind it. Two example invasions are shown, highlighted in yellow and green. The difference $\Delta P$ measures the absolute value of the change in the fluid pressure between the successive invasion of two pores. (b) Bursts are defined by record-breaking values of the pressure (here, highlighted in red). Their size is measured by the number of subsequent events required until the previous maximum pressure is exceeded. Forward and backward avalanches are defined, in contrast, for every invasion step (as in $[2,30]$ ). Their sizes, $S_{f}$ and $S_{b}$, give the number of invasion events required before that moment's invasion pressure is next exceeded, or was last seen, respectively.

throat apertures, and hence the invasion pressures. A simulation begins with a saturated pore space, and is invaded by air from one edge. The weakest throat exposed to the invasion line, or drying front, is invaded and the position of the front is adjusted accordingly. The weakest throat along the new front is then identified, the pore behind it invaded, and so on. An example of this algorithm is shown in Fig. 3(a).

The sequence of the throat invasions provides a sequence of invasion pressures, which can then be compared with experiments of similar, or identical, sample geometry. In this study, we consider the effect of spatial correlations on the dynamics of this pressure signal, $P$. Our quasi-static model is equivalent to the invasion-percolation problem with trapping $[1-3,34]$. This means that the further evolution of any isolated clusters, trapped behind the drying front, are not considered. Furthermore, the dynamics are tracked only by the sequence of pore invasions, rather than any more physically meaningful sense of time. In these ways it differs from a related pore-network model, which has been developed to explore the impact of 

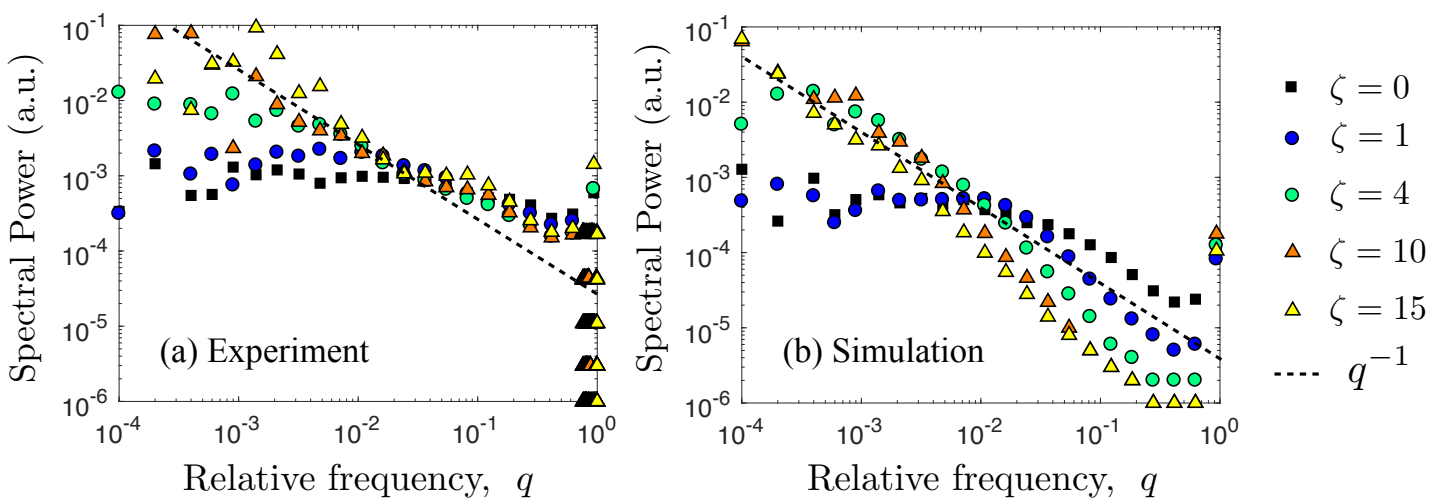

FIG. 4. The power spectra of the pressure signals from (a) experiments and (b) simulations respond to local correlations. For low frequencies, in particular representing fluctuations over tens or more invasion events, the uncorrelated porous media show relatively flat power spectra, characteristic of white noise. The introduction of correlations in the pore geometry adds significantly to the lowfrequency power, which corresponds to the jumps of the pressure shown in Fig. 2. For comparison, the dashed lines show a $1 / q$ response, which is classically associated with intermittency [15].

spatial correlations on drying rates [9]. The percolation model that we consider here exactly reproduces the sequence of pore invasions along the drying front of that pore-network model, but highlights the universal aspects of the system response.

\section{Burst and avalanche statistics}

We use a variety of statistics to characterise the fluctuations in the system pressure, $P_{i}$, over the sequence $i$ of pore invasions, as shown in Fig. 3(b). The power spectrum can be calculated directly from the pressure series, for a relative frequency $q$ normalised by the Nyquist or sampling rate, here that of the pore invasion rate. Between the invasion of any two successive pores there is a change in the invasion pressure of $\Delta P_{i}=\left|P_{i}-P_{i+1}\right|$, and the intermittency of the pressure signal can be characterised by the probability distribution of these steps, $D(\Delta P)$.

We also measure the burst size distribution $N(n)$, where $n$ is the size of a burst, defined as the number of events from one extreme value of the pressure, until this pressure is next exceeded. In other words, $n_{i}$ is the number of pores invaded between sequential recordbreaking values of the fluid pressure. For a fluid invasion experiment with a controlled 
injection pressure, invasion would be unstable between these points, and occur in a rapid 'burst', as in Refs. [4, 5]. In contrast, for drying [3, 20] or rate-controlled fluid drainage [34] the pore pressure relaxes during pore invasion, and the intermediate pressures can be more readily observed.

The number of record-breaking events, or bursts, in any particular experiment is relatively small. Therefore, to characterise the 'roughness' of the pressure fluctuations we also measure the size distributions of forward and backward avalanches, as defined in Refs. [2, 16, 30]. These note that after each and every pore invasion the pressure signal may take some time to recover to its former level, providing a local definition for the size of an invasion avalanche. In other words, each point in the pressure signal can be taken as the root, or starting point, of its own specific avalanche. The size of a forward avalanche, $S_{f}$, is thus defined as the number of pore invasion events required until the pressure at the root of that avalanche is next exceeded. Similarly, by reversing the time series, the size of a backward avalanche, $S_{b}$, may be calculated for every pore invasion, measuring the number of steps that have passed since that pressure at the root was last exceeded. For discrete data, these definitions are not necessarily equivalent, and can have different power-law distributions [30]. These local definitions of avalanches allow for all the arbitrarily small peaks and valleys of the pressure signal to contribute to a statistical distribution, rather than just the few extreme points representing the pressure bursts.

In the following results we will show how these metrics, and their distributions, are affected by correlated disorder in both experiments and simulations of invasion percolation.

\section{RESULTS}

The behaviours of fluids drying in correlated and uncorrelated random media are markedly different, as was shown in Fig. 2. These differences can be seen more clearly, especially in light of any experimental noise, in the power spectra of the pressure signals. As shown in Fig. 4, for uncorrelated $(\zeta=0)$ disorder, the low-frequency fluctuations in the pressure signal are comparable to white noise, for both experiment and simulation. As correlations are added, they add power to the low-frequency signal, increasing it until it approaches a $1 / q$ noise spectrum. Our aim here is to explain these differences, in light of the crossover length-scale provided by the correlation length of the disorder. 

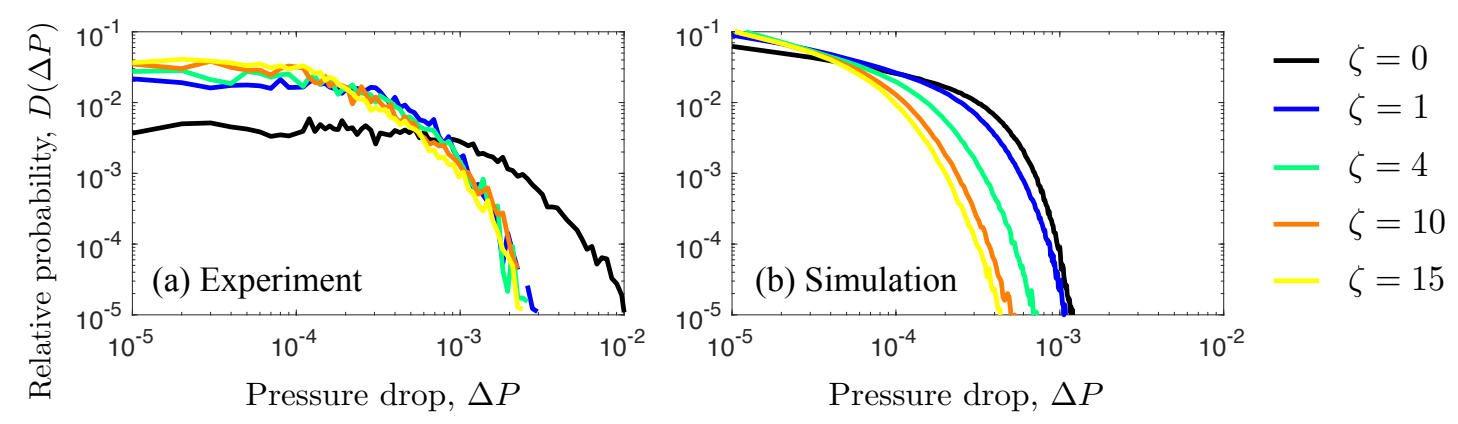

FIG. 5. Distributions of the pressure changes between subsequent pore invasions are shown for (a) drying experiments and (b) simulations. As the correlation length $\zeta$ increases, there is a tendency to have fewer extreme events, as more invasion events will occur within correlated regions of similar pore size. This is clearer in the simulation data, as uncertainties in exactly determining the experimental pressure signal introduce high-frequency random noise.

Correlations also affect the distribution of the pressure jumps between sequential invasion events, as represented by the high-frequency response of the invasion pressure. In Fig. 5 the probability distributions for the sizes of these jumps are plotted for both experiments and simulations. The plots have similar trends, depending on the correlation length $\zeta$. In particular, an increasing $\zeta$ implies that larger jumps become less common. This can be understood by noticing that once a pore has been invaded, it is likely to allow the drying front to explore an entire region made up of similarly-sized pores, with similar invasion pressures. The larger, rarer, pressure jumps will frequently represent the front travelling between correlated regions, separated by areas with tighter pores. Crossing such a highpressure barrier triggers an avalanche, and then the pressure will start to build up again as the front explores its new patch of available pores. Since the number of pores in a correlated region scales with $\zeta^{2}$, this sets a natural 'timescale' for considering crossover behaviour.

A common measurable of intermittent phenomena is the burst size distribution. As mentioned in the previous section, the number of bursts in any given experimental run is rather limited, as the number of record-breaking events typically grows logarithmically in time. Hence, we consider here only the simulation results, averaged over 60 independent realisations. To improve the statistics for this result we also simulate larger areas, of 700 $\times 700$ pores, and correlation lengths of up to $\zeta=30$. The results are presented in Fig. 6. Bursts in the uncorrelated $(\zeta=0)$ porous medium show a clear power-law behaviour, 


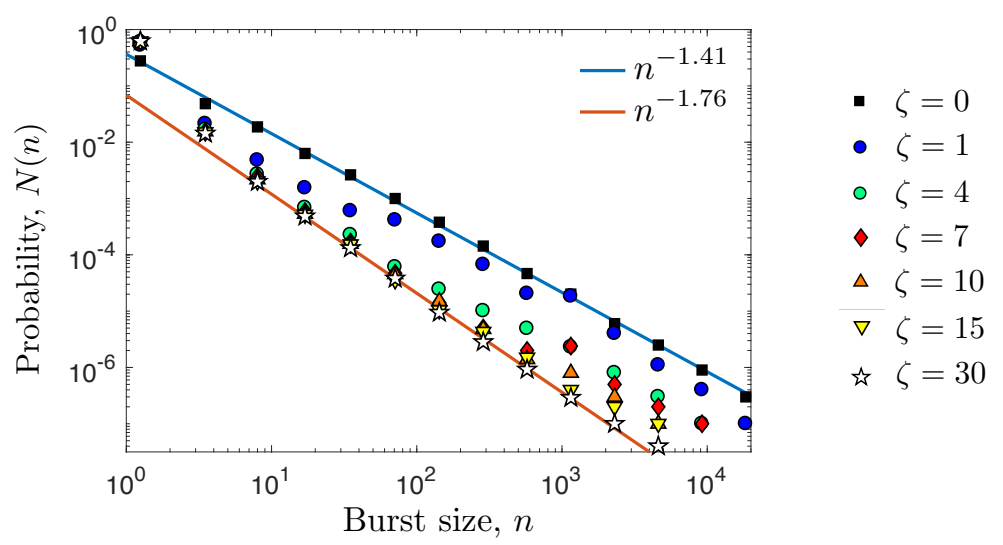

FIG. 6. A burst covers the invaded area between any two sequential record-breaking values of the saturation pressure. For pressure-controlled invasion, as in Refs. [4, 5], this would be a rapid 'burst' of activity between stable pressure values, while for our volume-controlled invasion it can be measured from the invasion pressure series, as in Fig. 3. In the absence of any correlations in pore size, the bursts follow a power-law distribution with an exponent that is similar to values reported for other examples of invasion percolation $[4,35,36]$. When correlations are introduced, the distribution shifts to a steeper power law, representing more frequent, but smaller, burst events, such as those confined to a single correlated patch of pores.

with $N \sim n^{-\tau}$. A least-squares fit to the logarithmically binned data gives an exponent of $\tau=1.41 \pm 0.03$. This agrees with recent experimental observations of comparable burst size statistics in drainage [4], where the exponent is measured at $1.37 \pm 0.08$. It is also close to, albeit slightly higher than, the burst size distribution predicted by percolation theory in an infinite $2 \mathrm{D}$ system [36], namely $17 / 13 \simeq 1.31$, and corresponding simulations [35]. However, as the correlation length $\zeta$ increases, the results shift away from this distribution, especially for smaller bursts. By $\zeta=30$ a large range of data is seen to be converging to a new power-law distribution, with the steeper exponent of $1.76 \pm 0.05$. For the intermediate correlation lengths, the events of smaller sizes resemble the highly correlated regime, while the larger events approach the response of the uncorrelated system. This crossover is rather intuitive, as the smaller events preferentially sample nearby pores with similar sizes, while larger events will be limited by pores outside the immediate correlated patch, which have more random fluctuations.

Avalanche size distributions, as defined in Fig. 3(b), offer a similar metric to bursts, but allow for more statistics to be gathered. They also show a crossover between two types of 

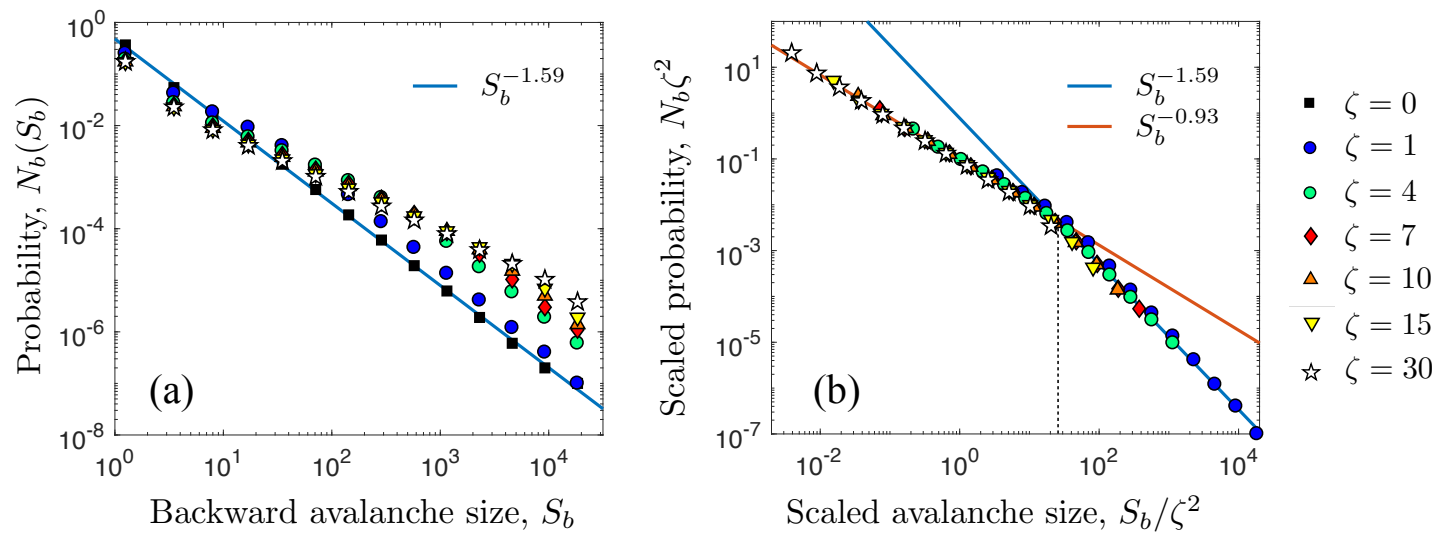

FIG. 7. Backward avalanches measure the number of events, going backwards in time, until the pressure of any particular invasion was last exceeded (see Fig. 3). The simulated distributions (a) collapse onto a master curve (b) when scaled by the area of a correlated patch of pores, $\zeta^{2}$. The crossover between these two scaling regimes, marked by a dashed line, occurs at around 5 times the correlation length, $\zeta$. Essentially, while small bursts are strongly affected by local correlations, events large enough to average over many such correlated patches show a mean-field distribution.

response. We consider the size of backward avalanches in Fig. 7, again for simulations of 700 $\times 700$ pores. For uncorrelated porous media, as in Fig. 7(a), the probability distribution of the backward avalanche sizes is a power-law, $N_{b} \sim S_{b}^{-\theta_{b}}$, with a critical exponent fit to $\theta_{b}=1.59 \pm 0.03$. As expected by the theory of invasion percolation [30], this value is related to the exponent of the burst size distribution by

$$
\theta_{b}=3-\tau
$$

For invasion into correlated porous media the avalanche size distribution also changes, and becomes shallower. When scaled by $\zeta^{2}$, as in Fig. 7(b), the results for all correlation lengths collapse onto one master curve with two distinct scaling regimes. Events that are significantly larger than the correlated area are consistent with the scaling of invasion percolation in uncorrelated porous media, and are fit by the exponent $\theta_{b}=1.59 \pm 0.06$. Smaller events, which are more likely to explore only a space confined to a single patch of similarly-sized pores, show a power-law distribution that is well-fit by the smaller exponent $0.93 \pm 0.03$. Interestingly, the relationship in Eq. 1 no longer seems to hold for these local events, where $\theta_{b}+\tau=2.69 \pm 0.06$. The crossover scale, estimated by the intersection of the two asymptotic curves, is about $5 \zeta$ (specifically, $\zeta^{2}=23.5$ ). This implies that avalanche events must cover 

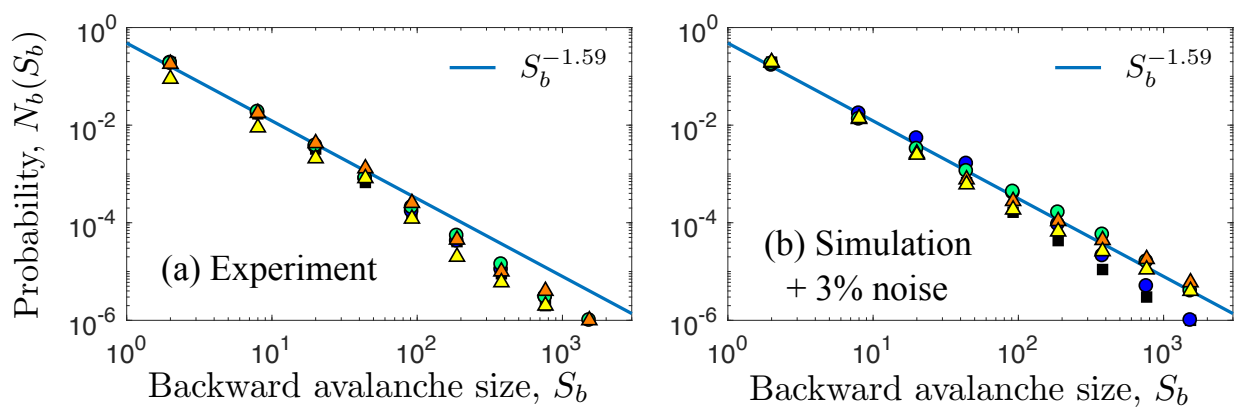

- $\zeta=0$

- $\zeta=1$

$\circ \zeta=4$

$\Delta \zeta=10$

$\Delta \zeta=15$

FIG. 8. Backward avalanches were measured in the experimental pressure signals. (a) There is no clear effect of correlation length in the avalanche statistics, despite its leading to intermittency in the original signal (Fig. 2) and power spectra (Fig. 4). We find, however, that the measured avalanche size distribution is strongly affected by noise, or uncertainties in the invasion pressure of each throat. (b) Adding 3\% random noise to each measurement, comparable to our experimental manufacturing tolerance [10], is enough to interfere with seeing the crossover behaviour of our simulations. As a guide to the eye, curves show the fit from Fig. 7 (a) for the noise-free $\zeta=0$ simulations.

a remarkably large area before they can average over enough local structure to return to a mean-field distribution.

We also measured backward avalanche sizes in the experimental invasion pressures, the distribution of which is given in Fig. 8(a). The data generally follow a power-law, which is consistent with the exponent of $\theta_{b}=1.59$ seen in the simulations of uncorrelated media. However, there is no noticeable effect of correlations on the experimental results, and specifically there is no clear crossover behaviour with $\zeta$. A possible reason for this discrepancy lies in experimental noise: there is a random manufacturing error, of about $\pm 3 \%$, in the pillar radii [10]. Since we rely on these radii to infer the pressure signal during drying, there will be a corresponding uncertainty in each pressure measurement. To test the implications of this, we added a $3 \%$ random perturbation to each value of the simulated pressure signals (using a $100 \times 100$ grid of pores, to match the experimental scale), and reanalysed the resulting data. As can be seen in Fig. 8(b), even this small uncertainty is sufficient to obscure the crossover between the two limiting types of distribution. In fact, the data from the simulations with noise now strongly resemble the experimental distributions.

Finally, we measured the forward avalanche sizes, and their distributions, in both sim- 


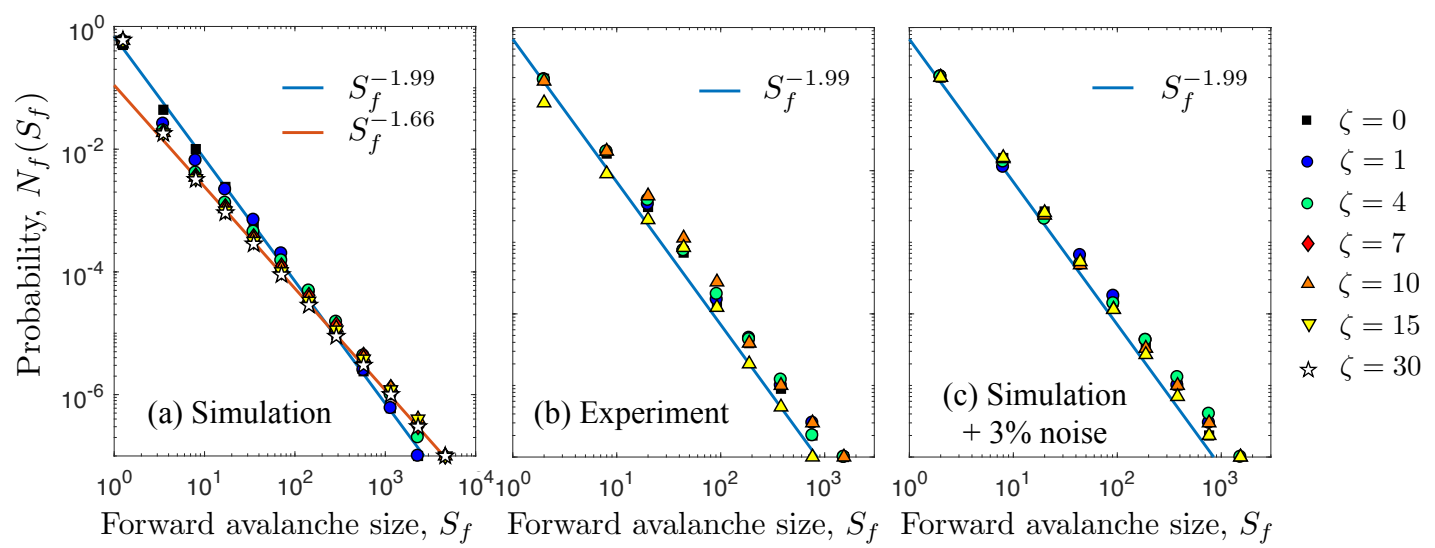

FIG. 9. Forward avalanches measure the number of events, going forward in time, until any particular invasion pressure is next exceeded (see Fig. 3). (a) In simulations the distribution for an uncorrelated porous medium follows a power-law distribution with exponent $\theta_{f}=1.99 \pm 0.05$. This is consistent with a 'superuniversal' distribution, where $\theta_{f}=2$, seen in a large class of invasion models $[16,30]$. The local correlations modify this distribution, lowering the exponent to $\theta_{f}=1.66 \pm 0.04$. (b) The forward avalanches in the experiment are show little variation with $\zeta$ and are consistent with $\theta_{f}=2$. (c) As with backward avalanches, the introduction of as little as $3 \%$ noise into the pressure signal interferes with the clear observation of the crossover behaviour.

ulations and experiments. The results, plotted in Fig. 9, show similar trends to those of backward avalanches. For simulations of invasion in uncorrelated porous media, and the larger grid of $700 \times 700$ pores, the probability distribution of forward avalanche sizes follows a power law with exponent $\theta_{f}=1.99 \pm 0.05$, as in Fig. 9(a). Correlated disorder modifies this exponent: for the $\zeta=30$ simulations, the data are instead well-fit by the lower exponent $\theta_{f}=1.66 \pm 0.04$, for example. However, again, this reduction in exponent cannot be detected either in the experimental data of Fig. 9(b), or in the $(100 \times 100$ pore $)$ simulations to which noise has been added to better mimic the experimental pressure signal, as shown in Fig. 9(c). In a large class of avalanche statistics, or intermittent phenomena, it is known that forward avalanches follow a 'superuniversal' distribution [16,30], with $\theta_{f}=2$, and our $\zeta=0$ results are consistent with that expectation. Interestingly, the local correlations in the geometry on which invasion happens are able to modify this critical exponent, with a crossover to a shallower exponent describing avalanches that are comparable or smaller to the area of a patch of similarly-sized pores. 


\section{DISCUSSIONS AND CONCLUSIONS}

Inspired by experimental observations of drying [9, 10], fluid-fluid invasion [6-8] and drainage $[4,5]$, we have investigated how local correlations in the structure of an otherwise random porous medium can affect how fluids move through that material. For this we used a micro-fluidic micro-model of drying, which allows matched numerical modelling, by either a pore-network model [9], or an invasion-percolation model of the leading drying front. In all cases, the introduction of even relatively weak local correlations in the size of nearby pores and throats visibly changed the sequence of invasion pressures. As represented by both time series (Fig. 2) and power spectra (Fig. 4), the local correlations increase the variability of the pressure signal. Specifically, they add to the low-frequency aspects of the pressure fluctuations, leading to a pattern similar to the $1 / f$ noise traditionally associated with intermittency.

These changes are due to the introduction of a length-scale, intermediate to the size of a single invaded pore, and the system size. It describes the size of a patch of similarly-sized pores, and corresponds to an invasion landscape consisting of easy-to-invade 'valleys', separated by more challenging 'peaks'. Such a geometry produces fewer extreme events, but adds longer-term fluctuations as activity shifts from one correlated region to another. These effects can be seen throughout our analysis, from the power spectra of Fig. 4, the distribution of pressure changes between sequential invasion events (Fig. 5), and the probability distribution of the sizes of pressure bursts (Fig. 6). Although it has been known that correlations in pore geometry can affect the breakthrough saturation and fractal dimension of invasion phenomena $[6,7,37]$, we have shown that they can also interfere with the expected relationships of the critical exponents of invasion problems. For example, when correlations are significant, the critical exponents of bursts and backward avalanches obtained in our model no longer sum to 3 , while that of forward avalanches is no longer required to be precisely 2 .

Surprisingly, very little correlation is required to modify the scaling laws of invasion percolation. We found a crossover between two sets of the critical exponents that characterise the burst size distribution (Fig. 6), and the distributions of backward (Fig. 7) and forward (Fig. 9) avalanches. Our geometry is constructed around an autocorrelation function $a=\exp (-\chi / \zeta)$, for two points separated by a distance $\chi$. Here, mean-field behaviour was 
recovered only for events spanning an equivalent scale of $\chi \geq 5 \zeta$, over which distance correlations will be virtually unmeasurable. For flows in real systems, such local correlations in material properties can be introduced in many ways. For example, by the roughness typical of a fracture surface $[38,39]$, or by a soil containing grains with a mixture of wettabilities [8, 40], or by pore-scale correlations that are present in even relatively uniform sedimentary rock like Berea sandstone [37]. One may expect, therefore, that the types of effects discussed here will apply to just as wide a range of situations. They also highlight the danger of coarse-graining, or continuum approximations, made without taking into account disorder, and fine-scale structure, in relation to percolation phenomena.

[1] David Wilkinson and Jorge F. Willemsen, "Invasion percolation: a new form of percolation theory," J. Phys. A: Math. Gen 16, 3365-3376 (1983).

[2] Stéphane Roux and Etienne Guyon, "Temporal development of invasion percolation," J. Phys. A: Math. Gen. 22, 3693-3705 (1989).

[3] Marc Prat, "Percolation model of drying under isothermal conditions in porous media," Int. J. Multiphas. Flow. 19, 691-704 (1993).

[4] Marcel Moura, Knut Jørgen Måløy, and Renaud Toussaint, "Critical behavior in porous media flow," EPL 118, 14004 (2017).

[5] Marcel Moura, Knut Jørgen Måløy, Eirik Grude Flekkøy, and Renaud Toussaint, "Verification of a dynamic scaling for the pair correlation function during the slow drainage of a porous medium," Phys. Rev. Lett. 119, 154503 (2017).

[6] M. A. Ioannidis, I. Chatzis, and E. A. Sudicky, "The effect of spatial correlations on the accessibility characteristics of three-dimensional cubic networks as related to drainage displacements in porous media," Water Resour. Res. 29, 1777-1785 (1993).

[7] Mark A. Knackstedt, Adrian P. Sheppard, and Muhammad Sahimi, "Pore network modelling of two-phase flow in porous rock: The effect of correlated heterogeneity," Adv. Water Resour. 24, 257-277 (2001).

[8] Julie Murison, Benoit Semin, Jean-Christophe Baret, Stephan Herminghaus, Matthias Schröter, and Martin Brinkmann, "Wetting heterogeneities in porous media control flow dissipation," Phys. Rev. Appl. 2, 034002 (2014). 
[9] Oshri Borgman, Paolo Fatinel, Wieland Lühder, Lucas Goehring, and Ran Holtzman, "Impact of spatially correlated pore-scale heterogeneity on drying porous media," Water Resour. Res. 53, 5645-5658 (2017).

[10] Paolo Fantinel, Oshri Borgman, Ran Holtzman, and Lucas Goehring, "Drying in a microfluidic chip: experiments and simulations," Sci. Rep. 7, 15572 (2017).

[11] Harihar Rajaram, Lin A. Ferrand, and Michael A. Celia, "Prediction of relative permeabilities for unconsolidated soils using pore-scale network models," Water Resour. Res. 33, 43-52 (1997).

[12] V. Mani and K. K. Mohanty, "Effect of pore-space spatial correlations on two-phase flow in porous media," J. Petrol. Sci. Eng. 23, 173-188 (1999).

[13] Muhammad Sahimi, Flow and transport in porous media and fractured rock, 2nd ed. (WileyVCH, 2011).

[14] Tom Bultreys, Wesley De Boever, and Veerle Cnudde, "Imaging and image-based fluid transport modeling at the pore scale in geological materials: A practical introduction to the current state-of-the-art," Earth-Sci. Rev. 155, 93-128 (2016).

[15] Per Bak, Chao Tang, and Kurt Wiesenfeld, "Self-organized criticality: An explanation of 1/f noise," Phys. Rev. Lett. 59, 381-384 (1987).

[16] Maya Paczuski, Sergei Maslov, and Per Bak, "Avalanche dynamics in evolution, growth, and depinning models," Phys. Rev. E 53, 414-443 (1996).

[17] James P. Sethna, Karin A. Dahmen, and Christopher R. Myers, "Crackling noise," Nature 410, 242-250 (2001).

[18] Hikaru Kawamura, Takahiro Hatano, Naoyuki Kato, Soumyajyoti Biswas, and Bikas K. Chakrabarti, "Statistical physics of fracture, friction, and earthquakes," Rev. Mod. Phys. 84, 839-884 (2012).

[19] William B. Haines, "Studies in the physical properties of soil. v. the hysteresis effect in capillary properties, and the modes of moisture distribution associated therewith," J. Agric. Sci. 20, 97-116 (1930).

[20] Lei Xu, Simon Davies, Andrew B. Schofield, and David A. Weitz, "Dynamics of drying in 3D porous media," Phys. Rev. Lett. 101, 094502 (2008).

[21] Marek Cieplak and Mark O. Robbins, "Dynamical transition in quasistatic fluid invasion of porous media," Phys. Rev. Lett. 60, 2042 (1988). 
[22] Ran Holtzman and Enrico Segre, "Wettability stabilizes fluid invasion into porous media via nonlocal, cooperative pore filling," Phys. Rev. Lett. 115, 164501 (2015).

[23] A. Sornette and D. Sornette, "Self-organized criticality and earthquakes," Europhys. Lett. 9, 197-202 (1989).

[24] D. V. Denisov, K. A. Lörincz, J. T. Uhl, K. A. Dahmen, and P. Schall, "Universality of slip avalanches in flowing granular matter," Nat. Comm. 7, 10641 (2016).

[25] Ken Tore Tallakstad, Renaud Toussaint, Stephane Santucci, and Knut Jørgen Måløy, "NonGaussian nature of fracture and the survival of fat-tail exponents," Phys. Rev. Lett. 110, 145501 (2013).

[26] Domingos S. P. Salazar and Giovani L. Vasconcelos, "Stochastic dynamical model of intermittency in fully developed turbulence," Phys. Rev. E 82, 047301 (2010).

[27] Xavier Gabaix, Parameswaran Gopikrishnan, Vasiliki Plerou, and H. Eugene Stanley, "A theory of power-law distributions in financial market fluctuations," Nature 423, 267-270 (2003).

[28] Ian Dobson, Benjamin A. Carreras, Vickie E. Lynch, and David E. Newman, "Complex systems analysis of series of blackouts: Cascading failure, critical points, and self-organization," Chaos 17, 026103 (2007).

[29] Per Bak and Kim Sneppen, "Punctuated equilibrium and criticality in a simple model of evolution," Phys. Rev. Lett. 71, 4083-4086 (1993).

[30] Sergei Maslov, "Time directed avalanches in invasion models," Phys. Rev. Lett. 74, 562 (1995).

[31] Nicos Martys, Marek Cieplak, and Mark O. Robbins, "Critical phenomena in fluid invasion of porous media," Phys. Rev. Lett. 66, 1058 (1991).

[32] Eyvind Aker, Knut Jørgen Måløy, Alex Hansen, and Soumen Basak, "Burst dynamics during drainage displacements in porous media: Simulations and experiments," EPL 51, 55 (2000).

[33] B. N. J. Persson, O. Albohr, U. Tartaglino, A. I. Volokitin, and E. Tosatti, "On the nature of surface roughness with application to contact mechanics, sealing, rubber friction and adhesion," J. Phys.: Condens. Matter 17, R1-R62 (2005).

[34] Knut Jørgen Måløy, Liv Furuberg, Jens Feder, and Torstein Jøssang, "Dynamics of slow drainage in porous media," Phys. Rev. Lett. 68, 2161-2164 (1992).

[35] Liv Furuberg, Knut Jørgen Måløy, and Jens Feder, "Intermittent behavior in slow drainage," Phys. Rev. E 53, 966-977 (1996).

[36] Nicos Martys, Mark O. Robbins, and Marek Cieplak, "Scaling relations for interface motion 
through disordered media: Application to two-dimensional fluid invasion," Phys. Rev. B 44, 12294 (1991).

[37] Mark A. Knackstedt, Adrian P. Sheppard, and W. V. Pinczewski, "Simulation of mercury porosimetry on correlated grids: Evidence for extended correlated heterogeneity at the pore scale in rocks," Phys. Rev. E 58, R6923-R6926 (1998).

[38] Jean Schmittbul, François Schmitt, and Christopher Scholz, "Scale invariance of crack surfaces," J. Geophys. Res 100, 5939-5973 (2011).

[39] Harold Auradou, Jean-Pierre Hulin, and Stéphane Roux, "Experimental study of miscible displacement fronts in rough self-affine fractures," Phys. Rev. E 63, 066306 (2001).

[40] Mina Bergstad and Nima Shokri, "Evaporation of $\mathrm{NaCl}$ solution from porous media with mixed wettability," Geophys. Res. Lett. 43, 4426-4432 (2016). 